\title{
Magmatismo granitoide arqueano e evolução geológica do Subdomínio de Transição da Província Carajás, sudeste do Cráton Amazônico, Brasil
}

Archean granitoid magmatism and geologic evolution of the Transition Subdomain of the Carajás Province, southeastern Amazonian craton, Brazil

\author{
Roberto Dall'Agnol \\ Universidade Federal do Pará/Instituto Tecnológico Vale
}

Davis Carvalho de Oliveira

Universidade Federal do Pará

Claudio Nery Lamarão

Universidade Federal do Pará

A Província Carajás é o mais importante domínio arqueano do Cráton Amazônico. Foi subdividida originalmente em três blocos, Cinturão Itacaiúnas, Terreno Granito-Greenstone de Rio Maria (TGGRM) e Cinturão Pau D'Arco (Araújo \& Maia, 1991; Costa et al., 1995), posteriormente reduzidos para dois, o TGGRM e o Bloco Carajás (Souza et al., 1996; Dall'Agnol et al., 1997, 2006; Althoff et al., 2000). O Bloco Carajás é constituído na sua porção norte pela Bacia Carajás e sua porção sul foi denominada informalmente de Domínio de Transição, interpretado como uma possível extensão do Domínio Rio Maria, afetada pelos eventos neoarqueanos que moldaram a Bacia de Carajás (Dall'Agnol et al., 1997, 2006). Mais recentemente, foi proposta para estes dois blocos a designação de domínios Rio Maria e Carajás (Vasquez et al., 2008). O Domínio Rio Maria, formado durante o final do Mesoarqueano (3,0 a 2,86 Ga), foi extensivamente estudado e os principais aspectos de sua evolução se encontram relativamente bem definidos, em particular no que diz respeito ao magmatismo granitoide arqueano (Macambira \& Lafon, 1995; Althoff et al., 2000; Souza et al., 2001; Dall'Agnol et al., 2006; Oliveira et al., 2009, 2011; Almeida et al., 2011, 2013).

A Bacia Carajás, formada no Neoarqueano sobre um substrato mesoarqueano, também foi alvo de muitos estudos, devido ao seu excepcional interesse metalogenético (Gibbs et al., 1986; DOCEGEO, 1988; Araújo et al., 1988; Machado et al., 1991; Teixeira \& Eggler, 1994; Pinheiro \& Holdsworth, 1997; Barros et al., 1997, 2009; Trendall et al., 1998; Lindenmayer et al., 2001; Tallarico et al., 2005; Lobato et al., 2005; Botelho et al., 2006). Porém, comparativamente ao TGGRM e à Bacia Carajás, a porção sul do Domínio Carajás, correspondente ao Subdomínio de Transição (Feio et al., 2013), até recentemente havia sido estudada apenas em nível de reconhecimento regional (Araújo \& Maia, 1991) e, apesar da relevante contribuição daqueles autores em termos de feições estruturais e evolução tectônica, havia um desconhecimento quase total em relação ao seu magmatismo granítico e idades das principais unidades, o que fazia com que os diferentes granitoides e gnaisses arqueanos fossem englobados indistintamente no Complexo Xingu.

Em razão disso, com o intuito de aprimorar substancialmente o quadro geológico existente, membros do Grupo de Pesquisa Petrologia de Granitoides (GPPG), do Instituto de Geociências da Universidade Federal do Pará (IG-UFPA) e da Faculdade de Geologia do campus de Marabá da UFPA, liderados pelos autores deste prefácio,

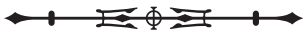


concentraram esforços nos últimos anos na realização de mapeamento geológico em escala 1:100.000 ou 1:50.000 e na caracterização e definição da idade dos principais granitoides arqueanos presentes no Subdomínio de Transição, de modo a contribuir para a compreensão de sua evolução e consequentemente da Província Carajás. Foram desenvolvidas no período, inseridas no Programa de Pós-graduação em Geologia e Geoquímica do IG-UFPA, dez dissertações de mestrado (ver os resumos das mais recentes no presente volume), sendo oito delas referentes ao Subdomínio de Transição e duas à porção norte do Domínio Rio Maria, e uma tese de doutorado, esta voltada para o estudo da área de Canaã dos Carajás na porção norte do Subdomínio de Transição, junto ao seu limite com a Bacia Carajás (Feio, 2011). Esta tese gerou a publicação de três artigos (Feio et al., 2012; Feio \& Dall'Agnol, 2012; Feio et al., 2013), que sintetizam os principais aspectos do magmatismo granitoide arqueano e a evolução no tempo daquele segmento da Província Carajás. Duas outras teses e diversas dissertações de mestrado estão em execução.

A intenção dos proponentes do presente dossiê publicado no Boletim do Museu Paraense Emilio Goeldi.

Ciências Naturais foi agregar contribuições representativas das pesquisas desenvolvidas no Subdomínio de Transição nos últimos anos pelos pesquisadores do GPPG. Outros artigos sobre esta temática estão sendo submetidos a este Boletim ou serão publicados em outros periódicos.

Constatou-se, a partir dos estudos realizados, em parte publicados nesta edição, que a porção leste do Subdomínio de Transição, que faz contato tectônico com o Cinturão Araguaia, é constituída dominantemente por associações de tonalitos e trondhjemitos com todas as características dos típicos TTG arqueanos. P. A. Santos et al. apresentam as características geológicas, petrográficas e geoquímicas das associações tonalítico-trondhjemíticas, presentes na porção leste do Subdomínio de Transição, a norte de Sapucaia, e discutem possíveis modelos petrogenéticos para explicar sua origem, estabelecendo comparações com associações afins da Província Carajás. Os tonalitos e trondhjemitos exibem altas razões $\mathrm{La} / \mathrm{Yb}$ e $\mathrm{Sr} / \mathrm{Y}$, tendo sido propostas para eles fontes à base de granada anfibolitos em altas pressões (ca. 1,5 GPa), com fracionamento de granada e, possivelmente, anfibólio. Possuem maiores similaridades com o Tonalito Mariazinha e o Trondhjemito Mogno, ambos do Domínio Rio Maria, e foram correlacionados com o Trondhjemito Colorado, descrito a oeste da área estudada por A. C. Silva et al. (comunicação pessoal, 2013). Abordam, ainda, rochas granodioríticas associadas às rochas mencionadas, mas concluem que não há vínculo genético entre granodioritos e tonalitos/trondhjemitos.

Por sua vez, M. F. B. Teixeira et al. concentraram suas pesquisas em leucogranodioritos e leucogranitos identificados nesse mesmo segmento do Subdomínio de Transição. Estudaram um corpo granodiorítico, ao qual denominaram de Leucogranodiorito Pantanal, com caráter peraluminoso, afinidade cálcio-alcalina, conteúdo relativamente elevado de Ba e Sr, padrões de ETR sem anomalias expressivas de Eu e com acentuado fracionamento de ETRP. Concluem que o magma granodiorítico não deve ser derivado da fusão parcial de fontes tonalítico-trondhjemíticas e que granada deve ter sido uma fase importante, seja no resíduo da fusão, seja durante o fracionamento do magma, ocorrendo o inverso com plagioclásio. Não descartam a hipótese de que os magmas granodioríticos possam resultar de interação entre magmas máficos hidratados derivados do manto (sanukitoides) e crosta ou líquidos tonalíticos. Uma variante desse modelo envolveria interação entre fluidos enriquecidos em K, Sr e Ba, derivados do manto metassomatizado, e rochas TTG antigas ou magmas TTG contemporâneos, modificados de modo a gerar os leucogranodioritos e granitos. Esta última hipótese foi a preferida pelos autores. O Leucogranodiorito Pantanal é cortado por leucogranitos deformados, com assinatura geoquímica de granitos tipo-A reduzidos, possivelmente correlacionáveis aos granitos da Suíte Planalto. 
M. J. P. Santos et al. focaram seu estudo na petrografia, geoquímica e geocronologia $\mathrm{Pb}-\mathrm{Pb}$ por evaporação em zircão de granitoides arqueanos encaixantes do batolito paleoproterozoico Seringa, também no Domínio Rio Maria e no limite com o Subdomínio de Transição. Distinguiram nesta área quatro tipos de granitoides arqueanos: 1) anfibólio-biotita-granodioritos com idade de cristalização de $2875 \pm 2$ Ma, conteúdo modal de máficos entre 16 e 21\%, teores elevados de Ni, Cr e Mg, correlacionados às rochas da Suíte Sanukitoide Rio Maria; 2) biotitagranodioritos com idade de cristalização de $2884 \pm 3$ Ma, distintos em termos petrográficos e geoquímicos das rochas anteriores, com proporções modais de minerais máficos variando de 8 a 15\%, conteúdo mais elevado de ETR e pronunciadas anomalias negativas de Eu; 3) leucogranodioritos e granitos, contendo fenocristais de plagioclásio e álcali-feldspato, correlacionados às rochas da Suíte Guarantã; 4) biotita-epidoto-trondhjemitos, com altas razões $\mathrm{La} / \mathrm{Yb}, \mathrm{Sr} / \mathrm{Y}$ e Nb/Ta e fortes similaridades com as rochas do Trondhjemito Mogno.

R. D. Santos et al. dedicaram-se à geologia, geoquímica e geocronologia do Diopsídio-Norito Pium em sua área-tipo. Este trabalho se reveste de grande importância devido à controvérsia sobre a origem metamórfica ou ígnea e a idade mesoarqueana ou neoarqueana desta unidade (Pidgeon et al., 2000; Ricci \& Carvalho, 2006; Vasquez et al., 2008; Galarza et al., 2012; Feio et al., 2012). Os dados obtidos pelos autores confirmam a idade neoarqueana (2,74 a 2,73 Ga) e as características ígneas da associação, constituída por noritos, gabronoritos, quartzo-gabros e enderbitos, que exibem localmente feições de magma mingling, sugerindo relações de contemporaneidade e cogeneticidade. A associação exibe caráter ferroso, natureza toleítica, afinidade geoquímica com basaltos intraplaca e teria sido derivada da fusão parcial de manto peridotítico. Dados isotópicos Sm-Nd forneceram idades-modelo $T_{D M}$ mesoarqueanas (3,14 a 3,06 Ga) e valores de $\varepsilon_{\mathrm{Nd}}(t=2,74 \mathrm{Ga}$ ) entre -2,78 e -1,58, indicando contribuição crustal em sua gênese.

Finalmente, C. Ronaib \& D. C. Oliveira estudaram a geologia da porção norte do TGGRM em área próxima do seu limite com o Subdomínio de Transição, situada a oeste de Xinguara e imediatamente a sul de Água Azul do Norte. Identificaram duas associações TTG distintas, as quais correlacionaram ao Tonalito Caracol e ao Trondhjemito Mogno, descritos originalmente em áreas próximas do TGGRM. Essas associações TTG são seccionadas por granodioritos e leucogranodioritos, cujos magmas teriam, segundo os autores, origem distinta daquela dos TTG. O biotita-granodiorito apresenta maior grau de deformação quando comparado com o leucogranodiorito e se distingue geoquimicamente dele por apresentar enriquecimento relativo em $\mathrm{Al}_{2} \mathrm{O}_{3}, \mathrm{CaO}$ e $\mathrm{Na}_{2} \mathrm{O}$, marcante anomalia positiva de Eu e padrão fortemente fracionado de ETRP, assim como ligeiro empobrecimento em $\mathrm{K}_{2} \mathrm{O}$. O controle composicional dessas rochas pode ter ocorrido em função de dois ou mais processos: (i) graus variáveis de fusão parcial de basaltos, sob diferentes níveis de pressão, o que controlaria a mineralogia residual predominante (em particular granada e feldspatos), exercendo forte controle no comportamento de ETRP, Al2 3 , da razão $\mathrm{K} 2 \mathrm{O} / \mathrm{Na} 2 \mathrm{O}$, Sr e Y; (ii) fonte mais enriquecida em sedimentos subductados da crosta oceânica, o que geraria magmas com composição similar ao biotita-granodiorito.

As informações obtidas nos trabalhos efetuados pelo GPPG no Subdomínio de Transição revelaram que ele não é homogêneo em termos de evolução geológica e tectônica e permitiram a distinção de dois novos domínios no mesmo, o domínio Canaã dos Carajás, situado ao norte e melhor caracterizado naquela área (Feio et al., 2013), e outro a sul, denominado de Domínio Sapucaia. Este novo modelo evolutivo foi divulgado durante o recente $13^{\circ}$ Simpósio de Geologia da Amazônia (Dall'Agnol et al., 2013) e um trabalho completo sobre o tema encontra-se em fase de preparação.

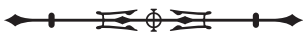


Acreditamos que o conjunto de artigos aqui publicado representa uma expressiva contribuição para o conhecimento geológico da Província Carajás, em particular do seu segmento conhecido na literatura como Subdomínio de Transição.

As pesquisas desenvolvidas receberam apoio do Instituto Nacional de Ciência e Tecnologia de Geociências da Amazônia (GEOCIAM; CNPq/MCT/FAPESPA - Processo n573733/2008-2), do Convênio VALE/FAP (Edital 01/2010 - ICAAF 053/2011), e representam uma contribuição para o projeto IGCP-SIDA-599.

\section{REFERÊNCIAS}

ALMEIDA, J. A. C., R. DALL'AGNOL, M. A. OliVEIRA, M. J. B. MACAMBIRA, M. M. PIMENTEL, O. T. RÄMÖ, F. V. GUIMARÃES \& A. A. S. LEITE, 2011. Zircon geochronology and geochemistry of the TTG suites of the Rio Maria granite-greenstone terrane: implications for the growth of the Archean crust of Carajás Province, Brazil. Precambrian Research 187(1-2): 201-221.

ALMEIDA, J. A. C., R. DALL'AGNOL \& A. A. S. LEITE, 2013. Geochemistry and zircon geochronology of the Archean granite suites of the Rio Maria granite-greenstone terrane, Carajás Province, Brazil. Journal of South American Earth Sciences 42: 103-126.

ALTHOFF, F. J., P. BARBEY \& A. M. BOULLIER, 2000. 2.8-3.0 Ga plutonism and deformation in the SE Amazonian craton: the Archean granitoids of Marajoara (Carajás Mineral Province, Brazil). Precambrian Research 104(3-4): 187-206.

ARAÚJO, O. J. B., R. G. N. MAIA, X. S. JORGE JOÃO \& J. B. S. COSTA, 1988. A megaestrutura arqueana da Folha Serra dos Carajás. Anais do Congresso Latino-Americano de Geologia 7: 324-338.

ARAÚJO, O. J. B. \& R. G. N. MAIA, 1991. Programa de levantamentos geológicos básicos do Brasil, Serra dos Carajás, folha SB-22Z-A, Estado do Pará. Texto explicativo: 1-164. DNPM/CPRM, Brasília.

BARROS, C. E. M., R. DALL'AGNOL, P. BARBEY \& A. M. BOULLIER, 1997. Geochemistry of the Estrela Grantite Complex, Carajás Region, Brazil: an example of an Archaean A-type granitoid. Journal of South-American Earth Sciences 10(3-4): 321-330.

BARROS, C. E. M., A. S. SARDINHA, J. P. O. BARBOSA \& M. J. B. MACAMBIRA, 2009. Structure, petrology, geochemistry and zircon $\mathrm{U} / \mathrm{Pb}$ and $\mathrm{Pb} / \mathrm{Pb}$ geochronology of the synkinematic Archean $(2.7 \mathrm{Ga}$ ) A-type granites from the Carajás Metallogenic Province, northern Brazil. Canadian Mineralogist 47(6): 1423-1440.

BOTELHO, N. F., M. A. MOURA, L. M. TEIXEIRA, G. R. OlivO, L. M. CUNHA \& M. U. SANTANA, 2006. Caracterização geológica e metalogenética do depósito de Cu \pm (Au, W, Mo, Sn) Breves, Carajás. In: O. J. MARINI, E. T. QUEIROZ \& B. W. RAMOS (Eds.): Caracterização de depósitos minerais em distritos mineiros da Amazônia: 335-390. DNPM, CT-Mineral/FINEP, ADIMB, Brasília.

COSTA, J. B. S., J. B. ARAÚJO, A. SANTOS, X. S. JORGE JOÃO, M. J. B. MACAMBIRA \& J.-M. LAFON, 1995. A Província Mineral de Carajás: aspectos tectono-estruturais, estratigráficos e geocronológicos. Boletim Museu Paraense Emílio Goeldi, série Ciências da Terra 7: 199-235.

DALL'AGNOL, R., Z. S. SOUZA, F. J. ALTHOFF, C. E. M. BARROS, A. A. S. LEITE \& X. S. JORGE JOÃO, 1997. General aspects of the granitogenesis of the Carajás metallogenic province. Extended Abstracts of the International Symposium on Granites and Associated Mineralizations 1: 135-161.

DAll'AgnOl, R., M. A. OliveirA, J. A. C. AlmeidA, F. J. AlthofF, A. A. S. Leite, D. C. OliveirA \& C. E. M. BARROS, 2006. Archean and Paleoproterozoic granitoids of the Carajás Metallogenetic Province, eastern Amazonian craton. Abstracts of Symposium on magmatism, crustal evolution, and metallogenesis of the Amazonian craton 1: 99-150.

DALL'AGNOL, R., D. C. OlivEIRA, F. V. GUIMARÃES, E. O. GABRIEL, G. R. L. FEIO, C. N. LAMARÃO, F. J. ALTHOFF, P. A. SANTOS, M. F. B. TEIXEIRA, A. C. SILVA, D. S. RODRIGUES, M. J. P. SANTOS, C. R. P. SILVA, R. D. SANTOS \& P. J. L. SANTOS, 2013. Geologia do Subdomínio de Transição do Domínio Carajás - implicações para a evolução arqueana da Província Carajás - Pará. Anais do Simpósio de Geologia da Amazônia 13: 1 CD-ROM.

FEIO, G. R. L. 2011. Magmatismo granitóide arqueano da área de Canaã dos Carajás: implicações para a evolução crustal da Província Carajás. Tese (Doutorado em Geologia) - Universidade Federal do Pará, Belém. Disponível em: <http://www.ppgg.ufpa.br/arquivos/ resumos/teses/2011_tese_Gilmara_Feio.pdf > . Acesso em: outubro 2013

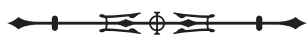


FEIO, G. R. L. \& R. DALL'AGNOL, 2012. Geochemistry and petrogenesis of the granites from the Canaã dos Carajás area, Carajás province, Brazil: implications for the origin of Archean granites. Lithos 154: 33-52.

FEIO, G. R. L., R. DALL'AGNOL, E. L. DANTAS, M. B. MACAMBIRA, A. C. B. GOMES, A. S. SARDINHA, D. C. OLIVEIRA, R. D. SANTOS \& P. A. SANTOS, 2012. Geochemistry, geochronology, and origin of the Neoarchean Planalto Granite suite, Carajás, Amazonian craton: A-type or hydrated charnockitic granites? Lithos 151: 57-73.

FEIO, G. R. L., R. DALL'AGNOL, E. L. DANTAS, M. J. B. MACAMBIRA, J. O. S. SANTOS, F. J. ALTHOFF \& J. E. B. SOARES, 2013. Archean granitoid magmatism in the Canaã dos Carajás area: implications for crustal evolution of the Carajás province, Amazonian craton, Brazil. Precambrian Research 227: 157-185.

GALARZA, M. A., D. C. OlIVEIRA, R. D. SANTOS, A. C. MARTINS, A. N. SANTOS \& P. J. L. SANTOS, 2012. Geocronologia do DiopsídioNorito Pium e granitóides associados, Canaã dos Carajás, Província Carajás. Anais do Congresso Brasileiro de Geologia 46: 1-CD-ROM.

GIBBS, A. K., K. R. WIRTH, W. K. HIRATA \& W. J. OLSZEWSKI JR., 1986. Age and composition of the Grão Pará Group volcanics, Serra dos Carajás. Revista Brasileira de Geociências 16: 201-211.

LINDENMAYER, Z. G., J. H. LAUX \& J. B. G. TEIXEIRA, 2001. Considerações sobre a origem das formações ferríferas da formação Carajás, Serra dos Carajás. Revista Brasileira de Geociências 31(1): 21-28.

LOBATO, L. M., C. A. ROSIÈRE, R. C. F. SILVA, M. ZUCCHETTI, F. J. BAARS, J. C. S. SEDANE, F. JAVIER RIOS, M. PIMENTEL, G. E. MENDES \& A. M. MONTEIRO, 2005. A mineralização hidrotermal de ferro da Província Mineral de Carajás - controle estrutural e contexto na evolução metalogenética da província. In: O. J. MARINI, E. T. QUEIROZ \& B. W. RAMOS (Eds.): Caracterização de depósitos minerais em distritos mineiros da Amazônia: 21-92. DNPM, CT-Mineral/FINEP, ADIMB, Brasília.

MACAMBIRA, M. J. B. \& J. M. LAFON, 1995. Geocronologia da Província Mineral de Carajás: síntese dos dados e novos desafios. Boletim do Museu Paraense Emílio Goeldi, série Ciências da Terra 7: 263-287.

MACHADO, N., Z. G. LINDENMAYER, T. E. KROGH \& D. LINDENMAYER, 1991. U/Pb geochronology of Archean magmatism and basement reactivation in the Carajás Área, Amazon Shield, Brazil. Precambrian Research 49(3-4): 329-354.

OLIVEIRA, M. A., R. DALL'AGNOL, F. J. ALTHOFF \& A. A. S. LEITE, 2009. Mesoarchean sanukitoid rocks of the Rio Maria Granite-Greenstone Terrane, Amazonian craton, Brazil. Journal of South American Earth Sciences 27(2-3): 146-160.

OLIVEIRA, M. A., R. DALL'AGNOL \& J. A. C. ALMEIDA, 2011. Petrology of the Mesoarchean Rio Maria suite and the discrimination of sanukitoid series. Lithos 127(1-2): 192-209.

PIDGEON, R. T., M. J. B. MACAMBIRA \& J. M. LAFON, 2000. Th-U-Pb isotopic systems and internal structures of complex zircons from an enderbite from the Pium Complex, Carajás Province, Brazil: evidence for the ages of granulites facies metamorphism and the protolith of the enderbite. Chemical Geology 166(1): 159-171.

PINHEIRO, R. V. L. \& R. E. HOLDSWORTH, 1997. Reactivation of Archean strike-slip fault systems, Amazon region, Brazil. Journal of the Geological Society 154: 99-103.

RICCI, P. S. F. \& M. A. CARVALHO, 2006. Rocks of the Pium-Area, Carajás Block, Brazil - a deep seated High-T Gabbroic Pluton (Charnockitoid-Like) with Xenoliths of Enderbitic Gneisses Dated at 3002 Ma - the basement problem revisited. Anais do Simpósio de Geologia da Amazônia 8: 1 CD-ROM.

RIO DOCE GEOLOGIA E MiNERAÇÃO S.A. (DOCEGEO), 1988. Revisão litoestratigráfica da Província Mineral de Carajás. Anais do Congresso Brasileiro de Geologia 35: 10-54.

SOUZA, S. Z., R. DALL'AGNOL, F. J. ALTHOFF, A. A. S. LEITE \& C. E. M. BARROS, 1996. Carajás Mineral Province: geological, geochronological and tectonic constrasts on the Archean evolution of the Rio Maria Granite-Greenstone Terrain and the Carajás block. Extended Abstracts of the Symposium on Archean Terranes of South America Platform 1: 31-32.

SOUZA, S. Z., H. POTREL, J. M. LAFON, F. J. ALTHOFF, M. M. PIMENTEL, R. DALL'AGNOL \& C. G. OLIVEIRA, 2001. Nd, Pb and Sr isotopes of the Identidade Belt, an Archaean greenstone belt of the Rio Maria region (Carajas Province, Brazil): implications for the Archaean geodynamic evolution of the Amazonian Craton. Precambrian Research 109(3-4): 293-315.

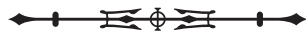


TALLARICO, F. H. B., B. R. FIGUEIREDO, D. I. GROVES, N. KOSITCIN, N. J. MCNAUGHTON, I. R. FLETCHER \& J. L. REGO, 2005. Geology and SHRIMP U-Pb geochronology of the Igarapé Bahia Deposit, Carajás Copper-Gold Belt, Brazil: an Archean (2.57 Ga) example of Iron-Oxide Cu-Au-(U-REE) Mineralization. Economic Geology 100(1): 7-28.

TEIXEIRA, J. B. G. \& D. H. EGGLER, 1994. Petrology, geochemistry, and tectonic setting of Archean basaltic and dioritic rocks from the N4 iron deposit, Serra dos Carajás, Pará, Brazil. Acta Geologica Leopoldensia 17: 71-114.

TRENDALL, A. F., M. A. S. BASEI, J. R. DE LAETER \& D. R. NELSON, 1998. SHRIMP zircon U-Pb constraints on the age of the Carajás formation, Grão Pará Group, Amazon Craton. Journal of South American Earth Sciences 11(3): 265-277.

VASQUEZ, M. L., L. T. ROSA-COSTA, C. G. SILVA, P. F. RICCI, J. O. BARBOSA, E. L. KLEIN, E. S. LOPES, E. B. MACAMBIRA, C. L. CHAVES, J. M. CARVALHO, J. G. OLIVEIRA, G. C. ANJOS \& H. R. SILVA, 2008. Geologia e recursos minerais do estado do Pará: Sistema de Informações Geográficas - SIG: Texto explicativo dos mapas Geológico e Tectônico e de Recursos Minerais do Estado do Pará. In: M. L. VASQUEZ \& L. T. ROSA-COSTA (Orgs.): Escala 1:1.000.000: 1-329. CPRM, Belém. 